\title{
Preisgekrönte Ekzemforscher
}

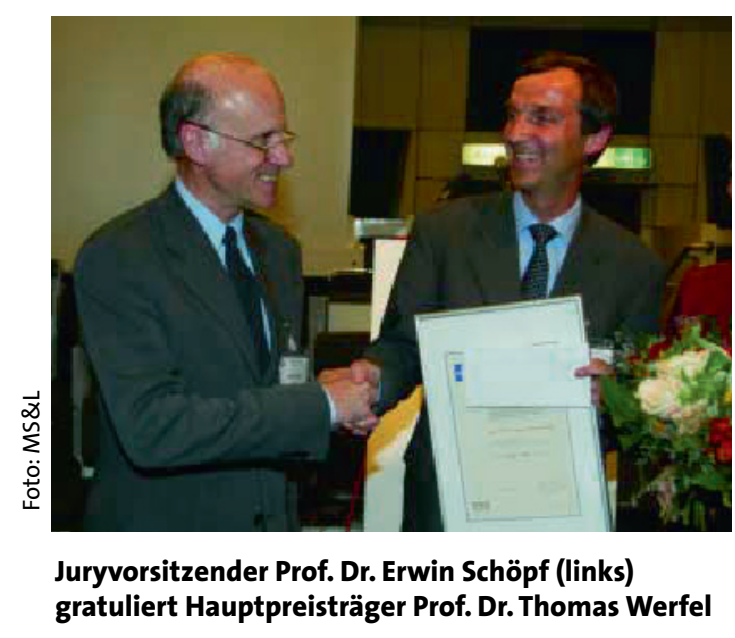

Die erstmalige Ausschreibung des Forschungspreises Immunmodulation durch Fujisawa in diesem Jahr führte zu einer Reihe hochkarätiger Bewerbungen. Die Jury vergab den Hauptpreis an die Arbeitsgruppe von Prof. Dr. Thomas Werfel.
D rof. Dr. Thomas Werfel von der Medizinischen Hochschule Hannover erhielt den Hauptpreis als Anerkennung seiner hervorragenden Forschungsarbeiten zum Verständnis der Immunmodulation in der Sparte Ekzemforschung. Dr. Suzan Artik von der Universitätshautklinik Düsseldorf und Dr. Stefan Martin von der Universitätshautklinik Freiburg erhielten jeweils Förderpreise.

„Prof. Werfel und seine Kollegen haben einen großartigen Beitrag zur Immunmodulationsforschung geleistet, indem sie unser Verständnis im diesjährigen Schwerpunkt ,Ekzemforschung' verbessert haben“, kommen- tierte Prof. Dr. Erwin Schöpf, der Vorsitzende der Jury. Er verlieh den mit $10.000 €$ dotierten Hauptpreis und die beiden mit $5.000 €$ dotierten Förderpreise im Rahmen des Kongresses der Deutschen Dermatologischen Gesellschaft in Berlin.

Die Vergabe des Forschungspreises Immunmodulation soll auf dem Gebiet der atopischen Dermatitis Arbeiten fördern, die zu einem besseren Verständnis der Immunmodulation beitragen. Das Unternehmen Fujisawa Deutschland will den Preis auch 2004 ausschreiben. Aktuelle Informationen dazu sind im Internet unter www.protopic.de abrufbar.

\section{Krefelder Hautschutzpreis}

Die Arbeitsgemeinschaft für Berufs- und Umweltdermatologie schreibt zur Förderung des Hautschutzes den „Krefelder Hautschutzpreis“ aus. Der mit $5.000 €$ dotierte Preis wird von der Degussa-Tochter Stockhausen $\mathrm{GmbH}$ \& Co. KG gestiftet. Der Krefelder Hautschutzpreis dient der Förderung von wissenschaftlichen Arbeiten auf dem Gebiet des beruflichen Hautschutzes und soll die interdisziplinäre Kooperation von Fachleuten in den Bereichen Berufsdermatologie, Arbeits- beziehungsweise Betriebsmedizin sowie Arbeitssicherheit würdigen.

Bewerber werden gebeten, ihre Arbeiten in fünffacher Ausfertigung in deutscher oder englischer Sprache bei der Arbeits- gemeinschaft für Berufs- und Umweltdermatologie einzureichen. Einsendeschluss ist der 28. Februar 2004 Die Preisverleihung findet im Rahmen des 7. Krefelder Hautschutztages am 22. Juni 2004 statt.

Weitere Informationen zum Hautschutztag und zum Hautschutzpreis sind online unter www.krefelder-hautschutztag.de abrufbar.

Arbeitsgemeinschaft für Berufs- und Umweltdermatologie

Prof. Dr. Peter Elsner

Klinik für Hautkrankheiten der

Friedrich-Schiller-Universität

Erfurter Straße 35

O7740 Jena 